Acta Crystallographica Section B

Structural

Science

ISSN 0108-7681

Editor: Carolyn P. Brock

\title{
Structure of a ferroelectric and ferroelastic monodomain crystal of the
} perovskite $\mathrm{BiFeO}_{3}$

\section{F. Kubel and H. Schmid}

This electronic document was scanned from an archival copy of material deposited to accompany a paper published in an IUCr journal. In many cases the only accessible copy was a microfilm of a poor-quality original. 
$2.11^{88}$

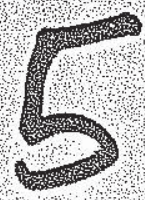

06.09 .90
1.

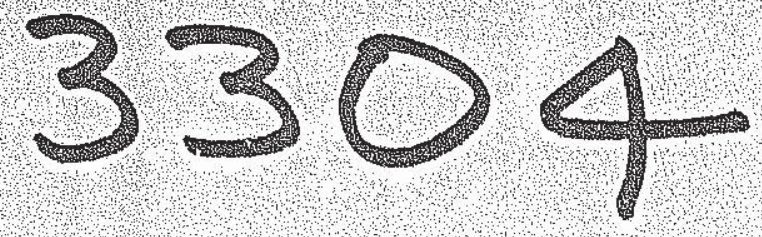

Acta Cryst. B

$L I S 7 I N G \quad F$

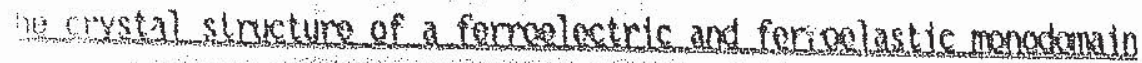
erustal of the darovkite Bifo03.

F Kulal and H.Semid

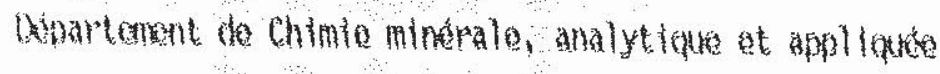

Universite de fontere,

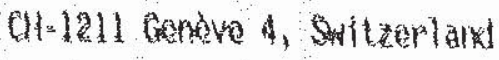




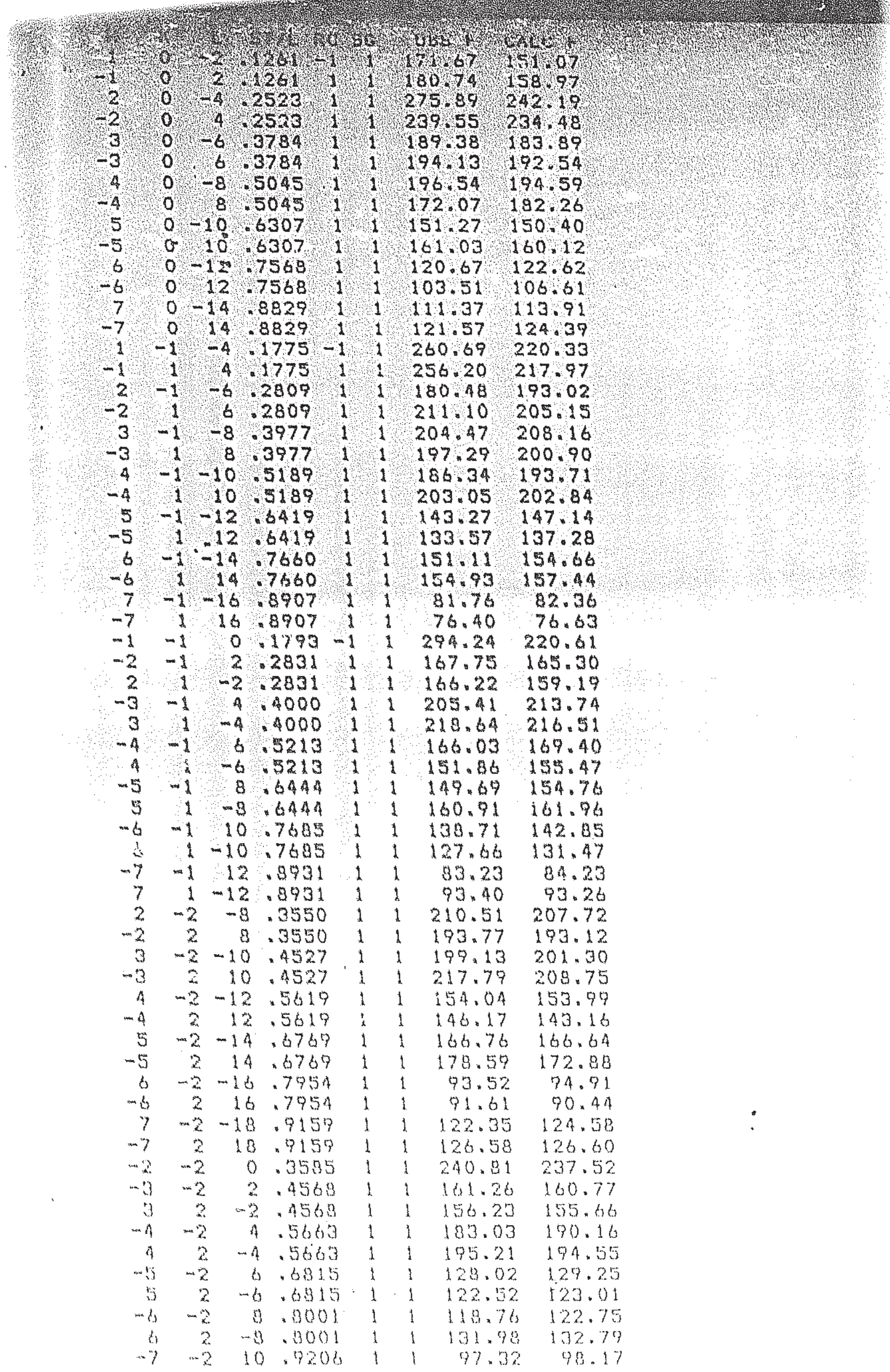




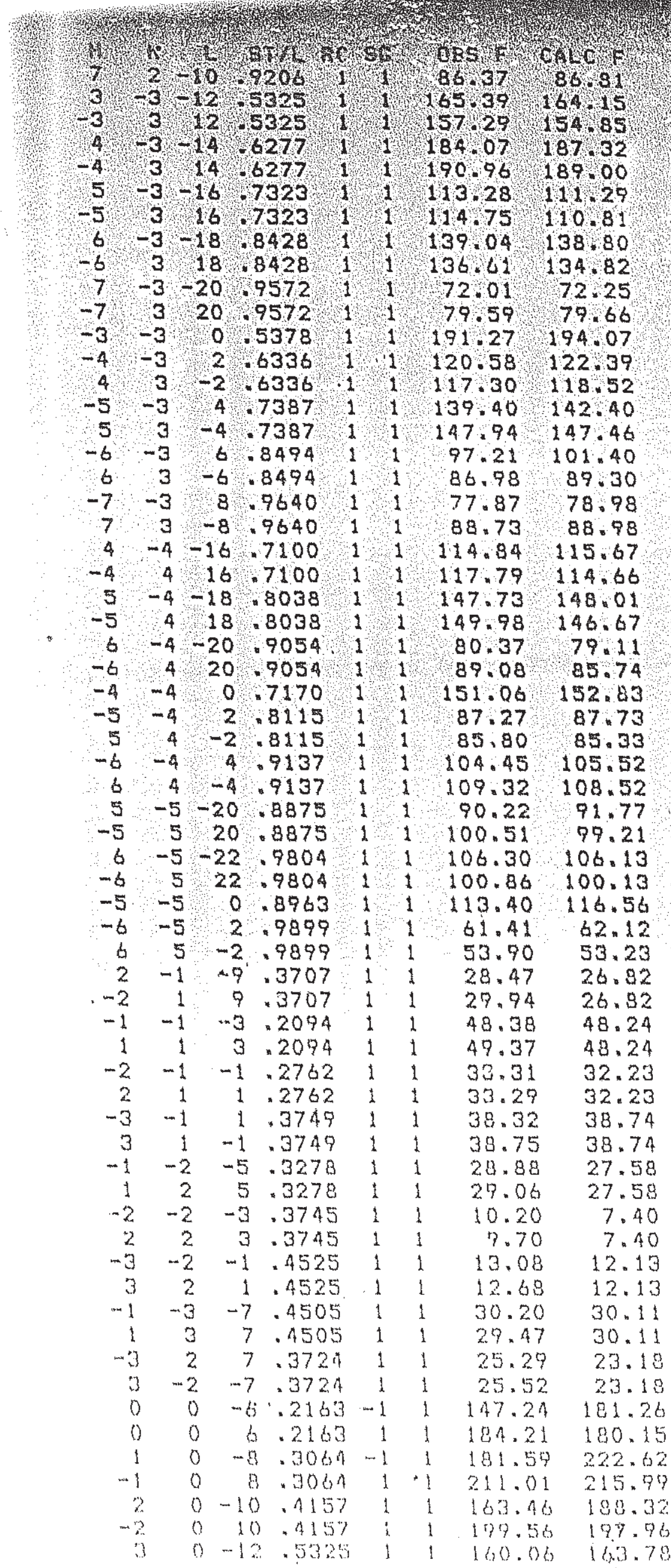




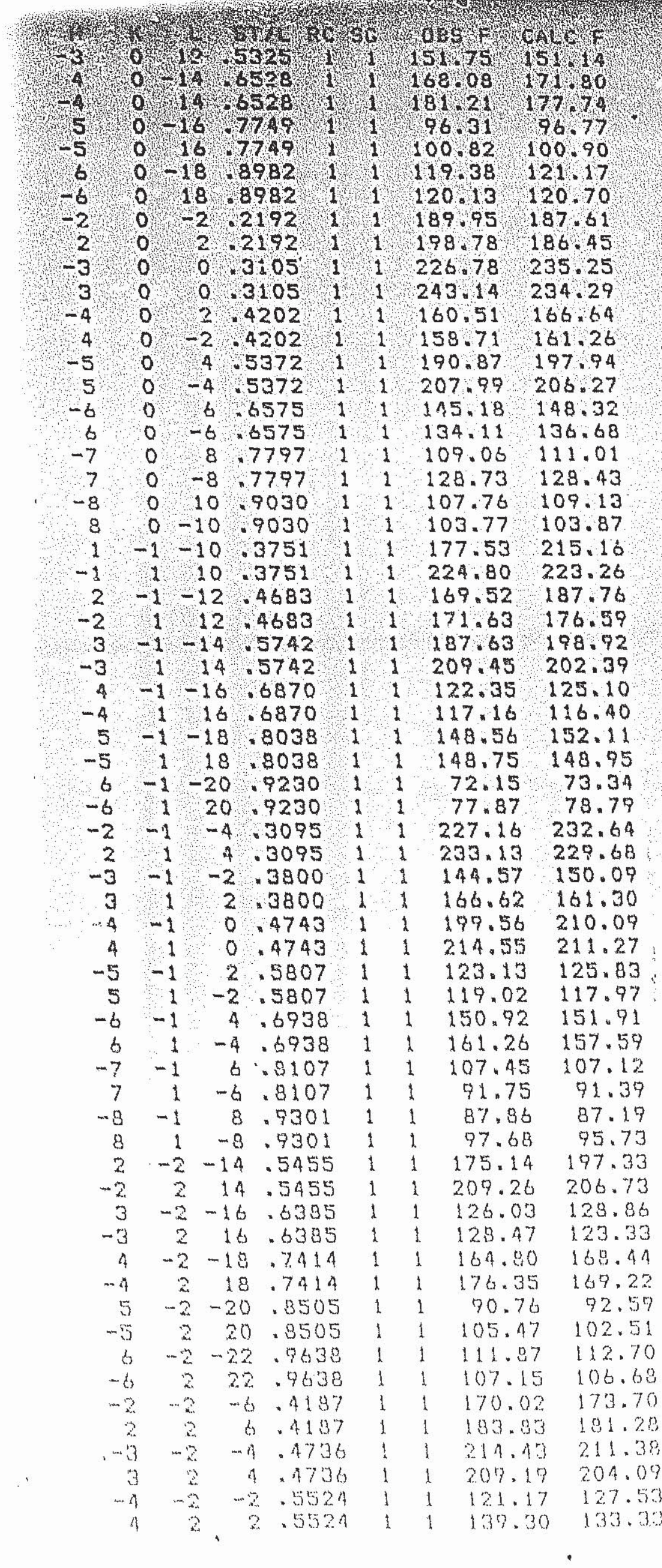




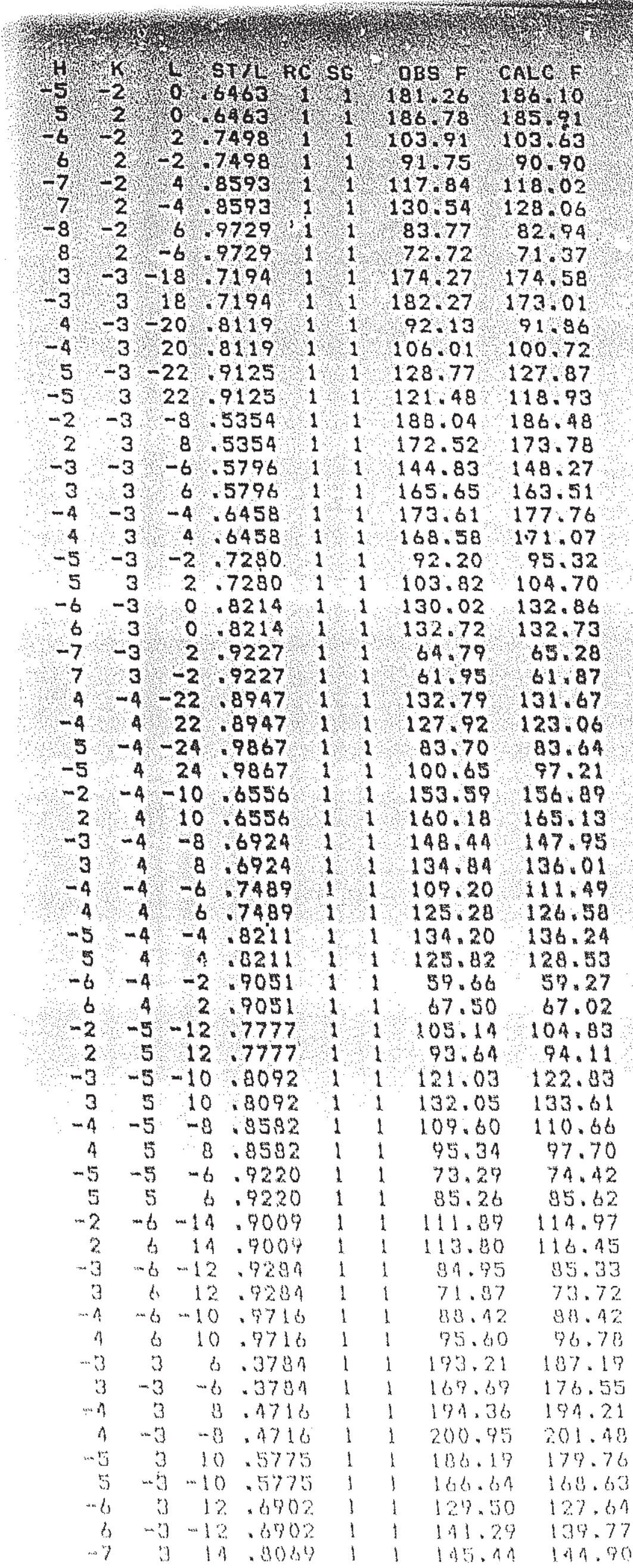




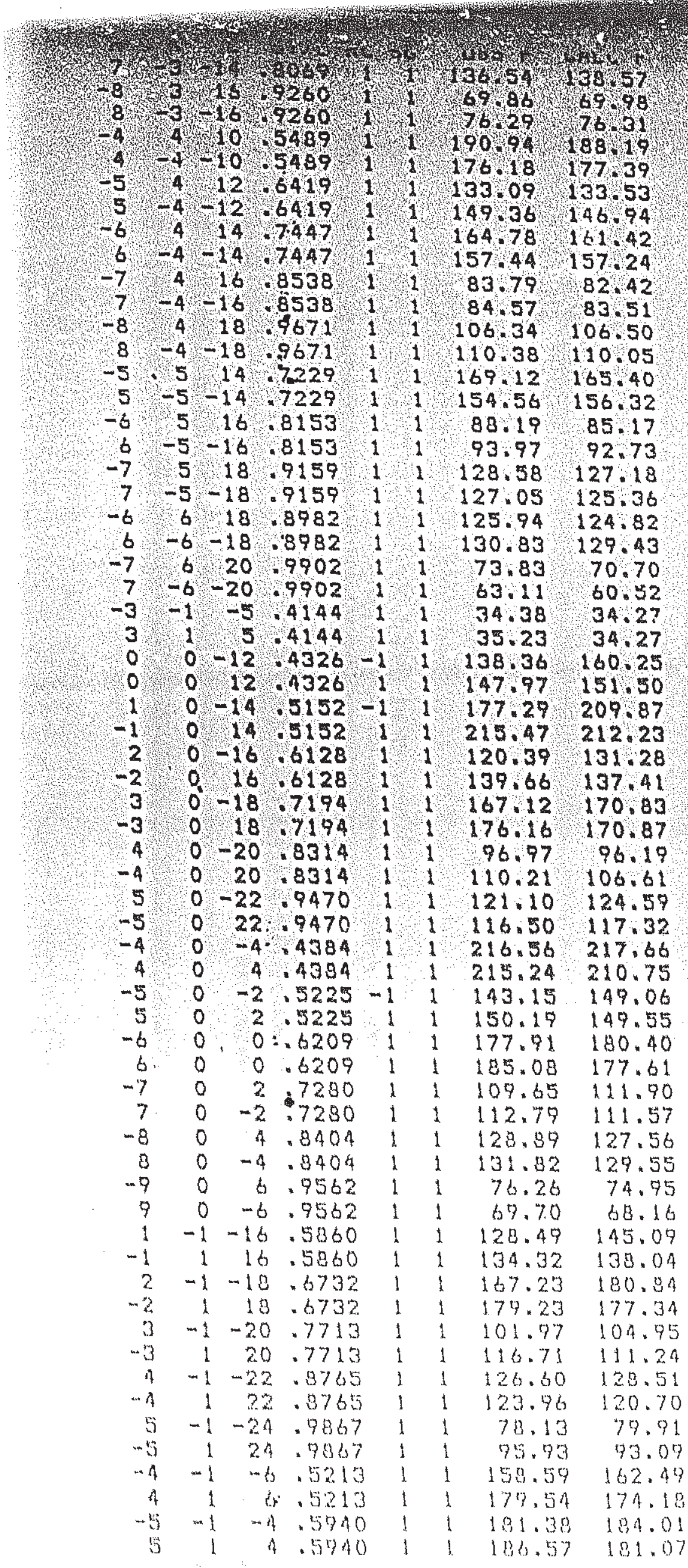




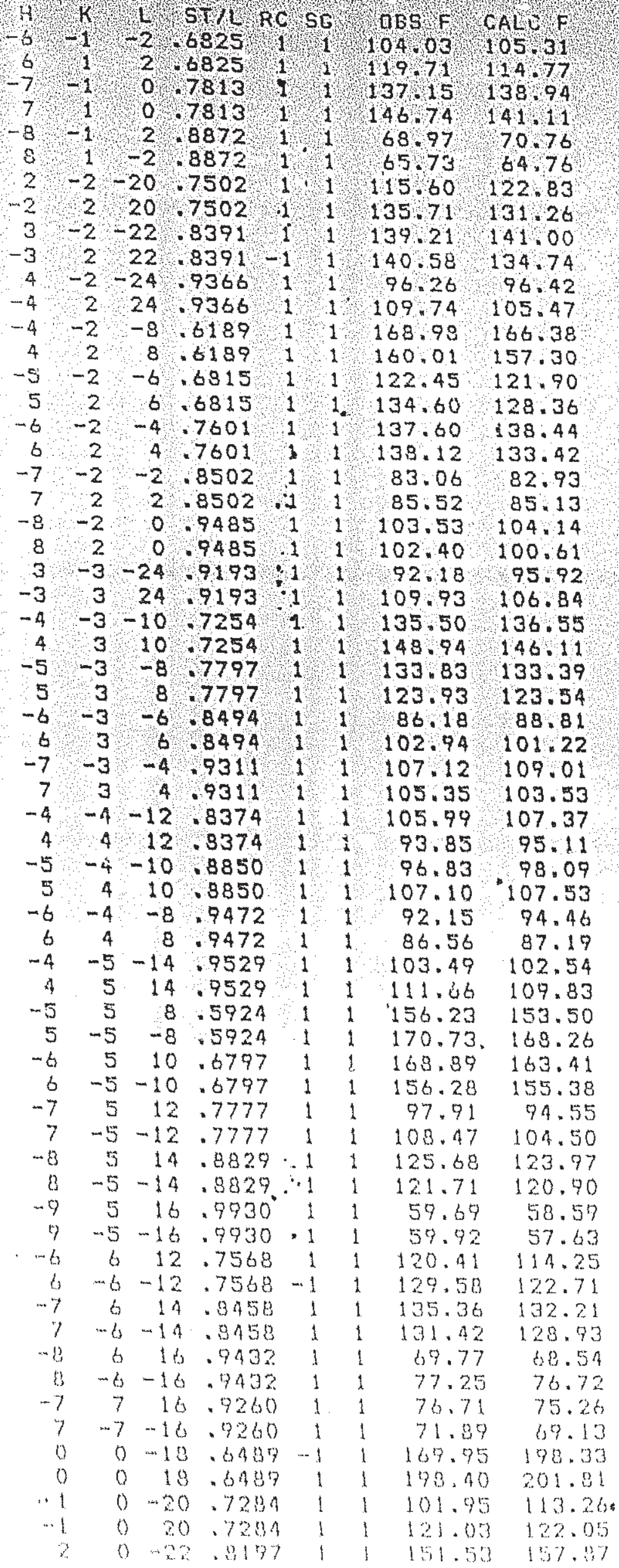




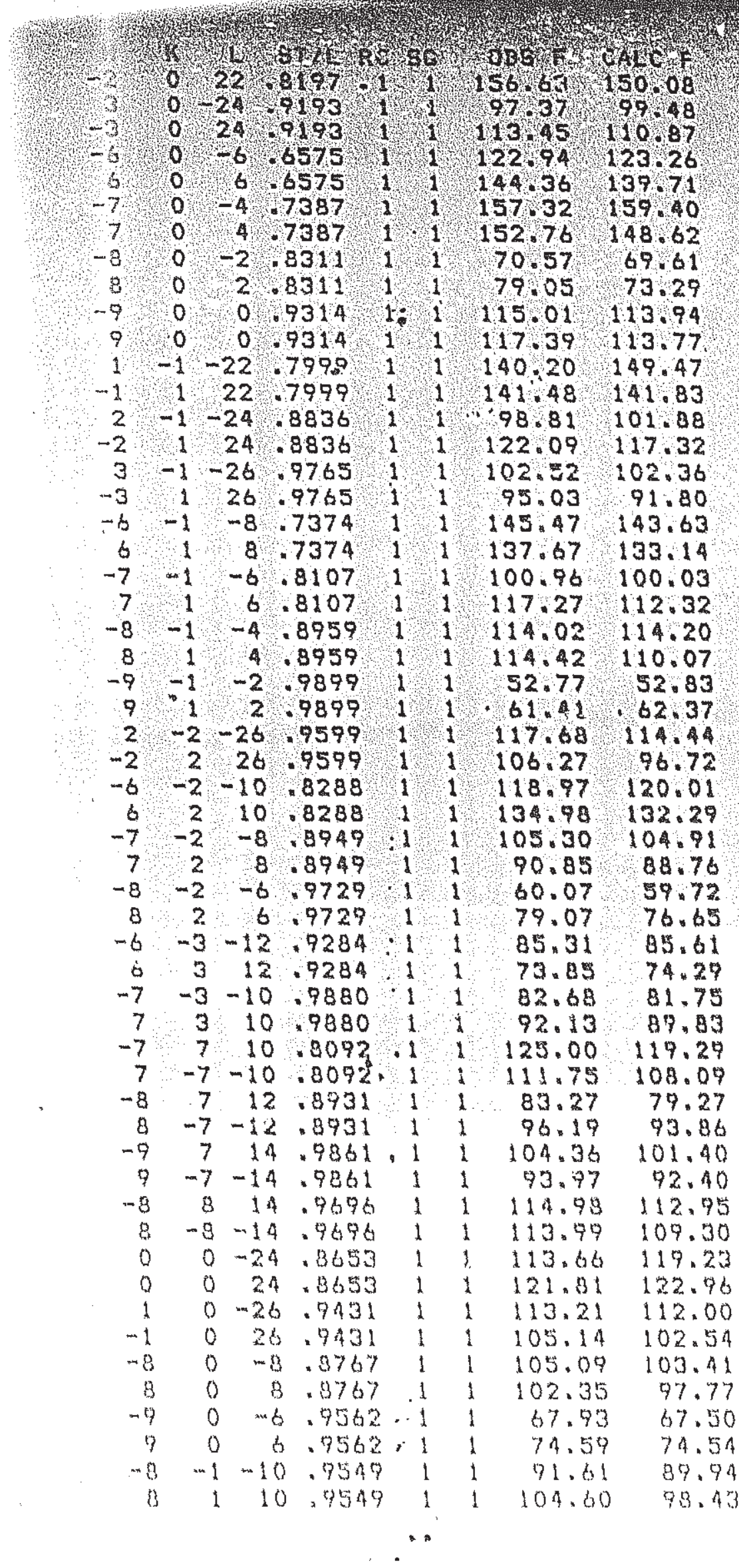




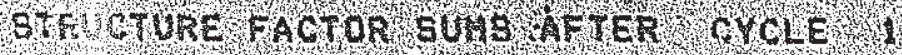

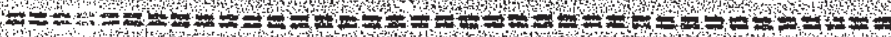

REFLCTIONS CONTRI EUTING TO LS MATRI $X$

SUM TOBSI

SUPG CALCI

SUM TOELTAI

SUM HETGHTADELTA*22

SORT (SUM (HEIGMT \&DELTAL\&?)/(NREF-NUAR)

R-FACTOR ( \ DELTA)

R-FACTOR (WW*DELTA)

R-FACTOR (WWDELTA*2)

NUMBER DF REFLECTIONS

NUABER OF YARTABLES

ALL REFLECTIONS

SUM/OBS,

SUSA /CALC/

SUM/DELTAI

SUA (WEIGHT\#DELTA*2)

SRRT (SUM (WEIGHT DELTA 2 )/ (NREF-NVAR))

\section{ALL REFL. \\ 53610.05 \\ 53534.11 \\ 1269.08 \\ 878,86 \\ 1.471}

.024

019

.023

422

16

ALL REFL.

55564.37

55504.94

1580.17

10308.73

4.972

.023

.028

.068

433

15
RCODE $=1$

53610.05

53534.11

1269.03

878.86

1.471

.024

.019

.023

422

RCODE 1

55564.37

55504.94

1580.17

10308.73

4.972

$\mathrm{RCODE}=2$

00

00

.00

100

.000

.000

.000

000

0

R-FACTOR ( DELTA)

R-FACTOR (WWDEELTA)

RMFATOR ( WDELTAW 2?

NUMBEF OF REFLECTIONS ?

NUABER OF VARIABLES

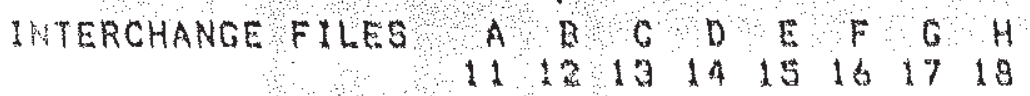

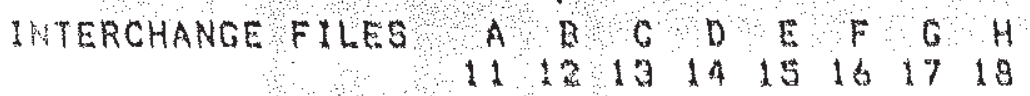

TIME OF DAY HH/MN/SS 15111145

HORDS OF MEMORY USED BY, GRYLSO 4844
CPU SECS CRYLSO

2.81000

MAXIMUM MEMORY USED THE RUN

QUMULATIUE OPU BEE a. 10000 5150

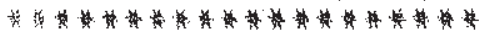

蕰 BEGIN FOURR

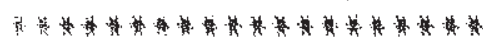

INPUT FILE FROM UNIT ID. FILE HISTORY.

SIFEO3 STARTX 15/2/89 10:34:11 ADDREF 15/2/89 10:34:13

LOADAY $15 /$ 2/89 10:30:39 LOADAT 15/2/89 10:40:27

CRYLSQ 15/ 2/89 10:40:39 LOADAT 15/2189 10:41:55

CRYLSQ 15/ 2/89 10:42:06 LOADAT 15/2/89 10:43:05

"RYLEA 15/ 2/89 10:43:17 LOADAT 15/2/89 10:45:07

CRYLSA 15/ 2/B9 10:45:21 LOADAT 15/ 2/89 10:47:21.

CRYLSQ $15 / 2 / 89$ 10:47:52 LOADAT 15/2/89 10:53:05

CRYLSQ 15/ 2/89 10:53:10 LOADAT 15/ 2/89 10:5.9:32

CRYLSQ $15 / 2 / 94$ 10:54:41 LOADAT 15/ $2 / 8914: 56: 42$

LOADAT 15/2/89 14:59:35 CRYLSQ 15/ 2/89 14:59:49

LOADAT 15/ 2/89 15:02:07 CAYLSQ $15 / 2 / 89$ 15:02:20

L.DADAT 15/ 2/89 15:09:0\& CRYLS日 15/ 2/89 $15: 09: 10$

LOADAT 15/ 2/89 15:11:39 CPYLS0 15/ 2/89 15:11:42 